\title{
Research on the application of hybrid cloud desktop design method
}

\author{
Hong WANG, Ya Jing XUE \\ College of Electronic and Electrical Engineering,Shanghai engineering technology university, Shanghai, China
}

\begin{abstract}
Through the study of hybrid cloud desktop , proposed technical solutions based on hybrid cloud desktop, On the basis of this, a detailed research and design of the hybrid cloud desktop is carried out, including the architecture of a hybrid cloud desktop solution and the PNS Desktop Virtualization (IDV), PNS Server Virtualization (VDI) and PNS QuickCloud cloud storage system integration, to achieve the centralized management and control of the client operating system, convenient and fast to meet the individual needs, centralized data management, the realization of the hybrid cloud desktop computer management, and improve the management level of the computer, improves the efficiency,it is virtual desktop changes to traditional PC desktop.
\end{abstract}

\section{Current status}

With the advance of information construction, The popularity of computer use Every year a large number of computers increase, installation and debugging software wasted a lot of manpower and material resources, it reduces efficiency, as a professional IT management, they hope to have a, the terminal is no need of maintenance and management, System and software installed on a server, it become managers of the dream scenario.

In daily work, computer viruses, data loss, application software error, system failure, some management problems have become the norm, these repetitive work invested a lot of manpower and occupied all the time. it has been unable to let managers again sufficient time to improve the construction of information.

At present, the mobile office will be a new office mode. In the process of daily office, mobile office demand is also increasing, office personnel in addition to the unit can handle the affairs and they can also in a local area network (LAN) of any piece of equipment, access to the unit of public resources, greatly improving work efficiency.

\section{Design objectives and features}

There are thousands of computers in schools, in view of the existing PC, they use IDV architecture to achieve a unified management of the virtual desktop, unified deployment, they improve management efficiency and reduce the failure rate. Virtual desktop to provide users with the current use of the PC as a variety of functions, they smooth use of the required software, smooth browsing web pages, and so on.

In view of the demand, we adopt the IDV virtual desktop and existing VDI, virtual desktop ,we can meet the needs of the existing management and teaching, the virtual desktop data of VDI andI IDV can be through a virtual storage to achieve interoperability.

IDV features: scalability, reliability, management

We use functional modularity. When the number of terminals or application requirements change, it can be easily extended by increasing the server, without the need 
to redesign the entire program. In this way, in order to maintain business continuity, not because the single point of failure affects the overall structure, resulting in the system is not available. The system has active monitoring service and alarm mechanism, which supports load sharing or fault switching mechanism, and realizes seamless transfer of load.

Through the management end can for the whole platform for unified management, including servers, virtual machines, virtual machine operating system, application software and network, etc.. The console can monitor the running state of the whole platform, including the server, the terminal and the network.

\section{Hybrid cloud virtual desktop Technology Architecture}

\subsection{Program architecture}

Hybrid cloud desktop virtualization topology includes three parts: VDI virtual desktop, IDV virtual desktop, virtual storage, it is a traditional PC desktop to the transformation of the virtual desktop

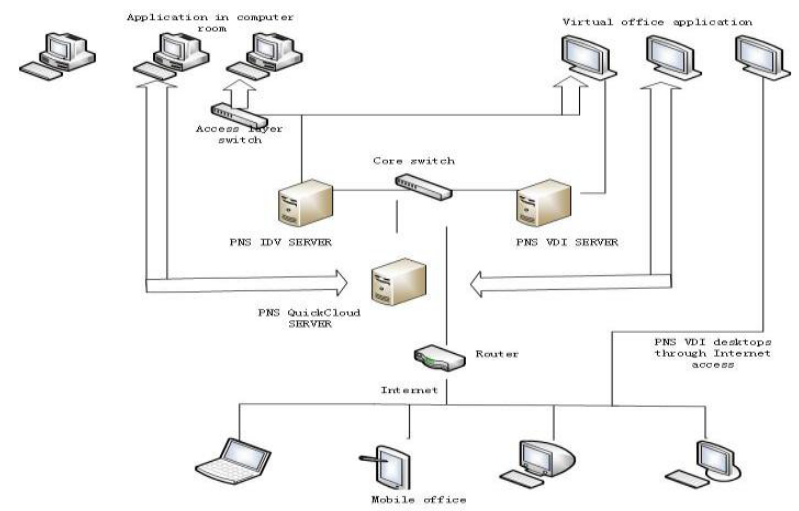

Figure 1. Program architecture

\section{Working principle :}

In the local area network, using the PNS desktop virtualization IDV server to push all kinds of operating system, software and services. In computer applications, have higher request for the client to use graphics performance, using common PC as the client, using PNS unique high performance local caching technology, it can fully play the PC local hardware performance, in order to meet the performance requirements.However, the client cannot access through the external network.

In need of remote access through the Internet to personal desktop, you can use PNS server virtualization VDI to create multiple virtual machine, virtual machine operating system unified by the PNS desktop virtualization push.. At this point, customers can use the PNS virtual client access to personal desktop through the Internet and telecommute.

Customer data are stored in the PNS cloud storage server.

Through the integration of PNS Desktop Virtualization (IDV), PNS Server Virtualization (VDI) and PNS cloud storage system, you can achieve:

- The client operating system centralized control

- Quick and easy to satisfy personalized needs

- Personal data of the centralized storage management .

- Internet remote office needs and remote data access requirements

- The local virtual desktop application of high performance

\subsection{Host characteristics}

1. Cloud desktop image (virtual desktop) system in the form of a sector is stored in operating system image

2. Desktop operating system in the form of data stream from the master mirror according to the need to transfer and run on the client terminal

3. With PNS HPLC technology will operating system cache in terminal local storage medium

4. The PNS OS over IP data link transmission protocol, in the network transmission is not affected by viruses and other cyber attacks and modifying the infection

5. Using TCP/IP based protocol, network transmission is not limited by network equipment features

6. The PNS Boot protocol and compatible with INTEL PXE protocol, they can unconditional across the gateway, routing, VLAN and VPN

\subsection{Storage cloud system}




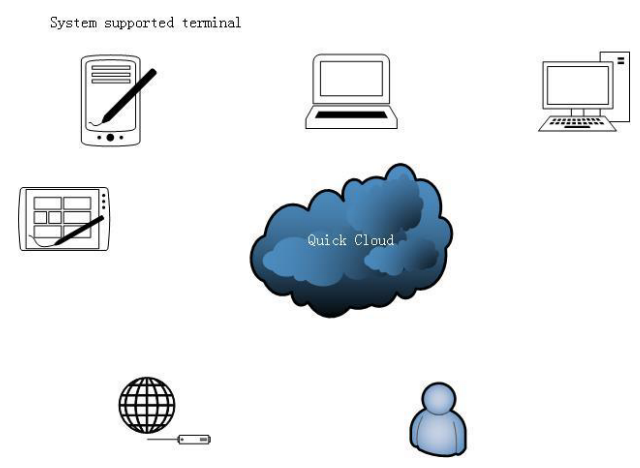

Figure 2. storage cloud system

QuickCloud PNS storage cloud system supports PNS High Performance Cloud desktop (virtual desktop) infrastructure, anytime, anywhere you can easily store data into the cloud. Cloud storage system to support multiple terminal (computers, mobile phones, ipad, etc.) unified storage, Make full use of existing idle resources, resource integration, unified management. With increasing capacity, performance and access speed of the linear expansion, providing similar drive mode of operation, in line with the day to day operating habits, physical storage group to realize the support power resume, FTP / SSH can direct transmission of large files, it can high and stable operation of security, Extensible security supervision mechanism, applicable toward secrecy demands a high level, extensible differential backup mechanism, ensure data security.

\subsection{PNS IDV virtual desktop}

PNS IDV virtual desktop uses is server network push mode, On the server side to install various user application system image, all the client uses the network boot mode and by server push operating system and service.

PNS desktop virtualization technology principles:

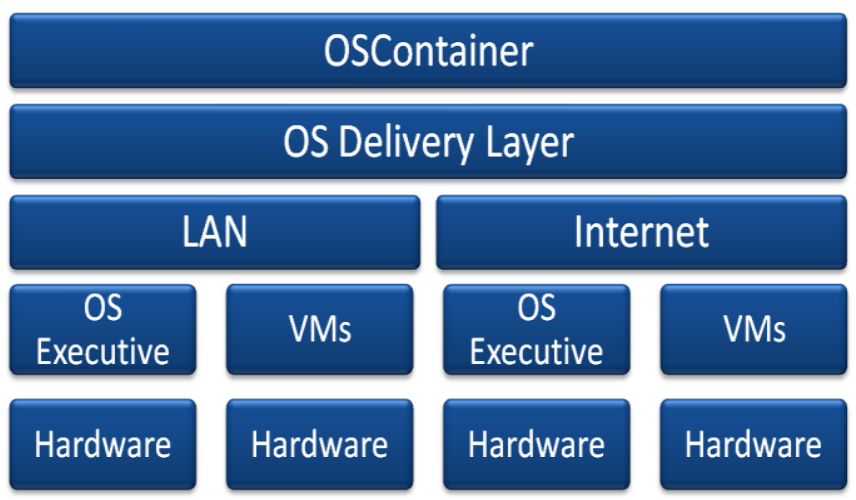

In the top is the operating system container

(OSContainer), in the container it can accommodate multiple operating system package, the operating system package by operating system push (OS delivery layer) system between the client and the server, network media can make local area network (LAN) or the Internet (Internet), operating system pushed to the client, by the operating system execution (OS) executive or virtual machine (VMS) execution, thereby driving the computer hardware.

\section{IDV desktop virtualization features:}

- Support load balancing

- can be flexibly expanded to support the virtual desktop in future

- Support Windows XP SP3/Windows 7/linux

- can remote management

\subsection{PNS VDI virtual desktop}

PNS server virtualization using VDI mode, you can create and use multiple virtual machines at the same time, Through the Internet remote access personal desktop in order to realize the remote office. This pattern and the combined desktop virtualization, all virtual client through desktop virtualization server push operating system and service, it has achieved a more centralized management.

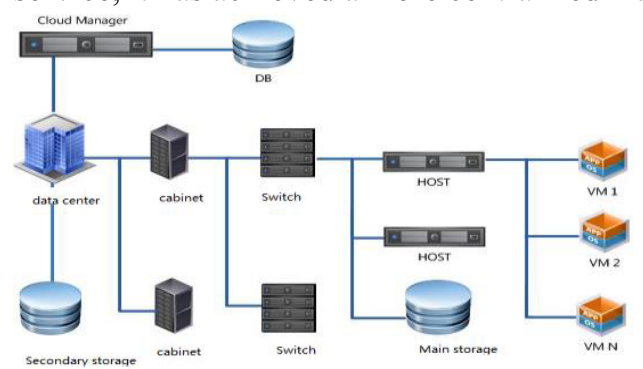

Figure 4. PNS VDI virtual desktop

VDI desktop virtualization features:

according to different application scenarios, the corresponding hardware configuration server, and unified management by the virtualization platform.

The centralized storage scheme, to ensure high availability and load balancing.

We need to consider in late stage development, hardware and software are able to support smooth expansion.

Figure 3. PNS desktop virtualization technology rinciples 


\subsection{PNS cloud storage system}

Personal data are centrally stored by the PNS, you can access a variety of terminal equipment through the LAN or Internet .

Virtual storage characteristics:

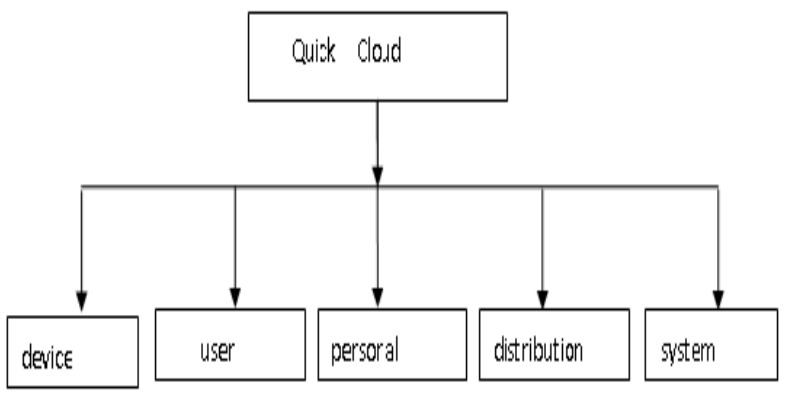

Figure 5. PNS cloud storage system

- can browse data by PC, ipad, mobile phone

- The virtual memory can be mapped into the letter in the use of virtual desktop;

\section{Summary}

For schools, computing center is a school for the students practice, is representative of the image of the school, building based on cloud desktop virtualization is a great revolution to the traditional desktop management, For the future schools has important significance for the development of computer technology.

\section{References}

1. Wang Qingbo, why virtualization and cloud computing $[\mathrm{M}] .28-39$. Electronic Industry Press.

2. stone Yirong, Duan Yong. Cloud computing in the field of application of Telecom Telecom I T science, 2009,25 (9).

3. Zhu Jinzhi wisdom of the cloud computing [M].68-108. Electronics Industry Press.

4. Liu Peng. Cloud computing [M].47-89. electronics industry publishing Press.

5. Wang Feng, Jiang Feng, Li Zhaoyang. Analysis of the virtual desktop and key technology. Telecom technology, 2011,1 (8).

6. Tao Caixia, Chen Kang. Cloud computing in telecom support system in the field of application and analysis, telecommunication science, 2010, 26 (10) (deadline: 2011-05-27) based on Virtualization cloud desktop technology program research and design of communication hot) 